\title{
Concomitant medications and clinical outcomes in idiopathic pulmonary fibrosis
}

\author{
To the Editor:
}

Patients with idiopathic pulmonary fibrosis (IPF) frequently have a substantial burden of comorbidities [1]. Antifibrotic therapy is recommended to slow the progression of IPF [2]. Patients receiving antifibrotic therapy frequently receive concomitant medications for the management of comorbidities [1, 3-9]. Previous post hoc analyses of antacids, statins, metformin, anticoagulants and angiotensin modulators in patients with IPF enrolled in phase III randomised controlled trials (RCTs) have generated hypotheses on the impact of these treatments on IPF outcomes [3-9]. The effects of multiple concomitant medications in patients with IPF have been largely unexplored. The objective of the present analyses was to explore the association between use of combinations of frequently prescribed concomitant medications and disease outcomes in patients with IPF.

Patients who received placebo in ASCEND (study 016; NCT01366209) and CAPACITY (studies 004 and 006; NCT00287716 and NCT00287729) and patients randomised to receive placebo or interferon- $\gamma-1 \mathrm{~b}$ in INSPIRE (NCT00075998; no treatment effect was observed) were included in the present analyses [10-12]. Eligibility criteria and data collection have been described previously [10-12]. Baseline medication use was characterised by the drug/drug class and number of drug/drug classes patients were receiving. Drug/drug classes of interest were selected based on the number of patients and effects seen in previous analyses and pre-analyses [3-7]. The outcome was a composite end-point of disease progression, defined as the first occurrence of absolute decline in forced vital capacity (FVC) $\geqslant 10 \%$ predicted, decline in 6 -min walk distance $(6 \mathrm{MWD}) \geqslant 50 \mathrm{~m}$ or death from any cause over 52 weeks. This composite end-point was evaluated in ASCEND and in previous post hoc analyses of medication use in pooled data from ASCEND and CAPACITY [3-7, 10]. Associations between baseline medication use and the study outcome were estimated using Cox proportional hazard models; hazard ratios (HRs) were adjusted for age, sex, smoking status, baseline physiological function (FVC \% pred and diffusing capacity of the lung for carbon monoxide \% pred), 6MWD, University of California, San Diego Shortness of Breath Questionnaire and comorbidities, which were selected for inclusion using the stepwise method. In the models, medication use was characterised using two independent binary variables and the pairwise combination of the two binary variables. Patients with missing baseline information were excluded from multivariable analyses.

The full analysis population comprised 1450 patients with IPF. At baseline, the most frequently reported concomitant medications were proton pump inhibitors (PPIs) ( $\mathrm{n}=604,41.7 \%$ ), antithrombotics (including anti-aggregants) $(n=604,41.7 \%)$, statins $(n=568,39.2 \%)$, obstructive airway medications $(n=497,34.2 \%)$ and anti-inflammatory medications $(n=423,29.2 \%)$. Few patients were receiving these medications alone (without at least one concomitant medication) (PPIs $n=54,3.7 \%$; antithrombotics $n=10,0.7 \%$; statins $\mathrm{n}=13,0.9 \%$; obstructive airway medications $\mathrm{n}=60,4.1 \%$; anti-inflammatory medications $\mathrm{n}=33,2.3 \%$ ). At baseline, $153(10.6 \%)$ patients were receiving no medication, while 754 (52.0\%) were receiving between one and three medications and $543(37.4 \%)$ were receiving four or more medications. The most frequent pairwise combinations of medications (with or without additional concomitant medications) were antithrombotics and statins ( $\mathrm{n}=367,25.3 \%)$, PPIs and antithrombotics $(\mathrm{n}=298,20.6 \%)$ and PPIs and statins $(n=273,18.8 \%)$. At baseline, 77 unique combinations of medications were reported in two (10.6\%) patients each, and 342 unique combinations were reported in one (23.6\%) patient each.

@ERSpublications

This post hoc exploratory analysis found no clear associations between frequently used concomitant medication combinations and disease progression in 1450 patients with IPF enrolled in phase III trials, but several combinations may require further study. http://bit.ly/2ZzyMXR

Cite this article as: Kreuter M, Lederer DJ, Cottin V, et al. Concomitant medications and clinical outcomes in idiopathic pulmonary fibrosis. Eur Respir J 2019; 54: 1901188 [https://doi.org/10.1183/ 13993003.01188-2019]. 
At baseline, the most frequently reported comorbidities were hypertension ( $n=757,52.2 \%)$, obesity $(\mathrm{n}=616,42.5 \%)$, hypercholesterolaemia $(\mathrm{n}=556,38.3 \%)$, cardiovascular disease (CVD) $(\mathrm{n}=386,26.6 \%)$, gastro-oesophageal reflux disease (GORD) $(n=325,22.4 \%)$ and diabetes $(n=304,21.0 \%)$. However, few patients reported these comorbidities alone (without at least one additional comorbidity; hypertension $n=79,5.5 \%$; obesity $n=92,6.3 \%$; hypercholesterolaemia $n=48,3.3 \%$; CVD $n=15,1.0 \%$; GORD $n=32,2.2 \%$; diabetes $n=17,1.2 \%)$. Only $202(13.9 \%)$ patients reported no comorbidities, while 23 unique combinations of comorbidities were reported in two $(3.2 \%)$ patients each, and 118 unique combinations were reported in one $(8.1 \%)$ patient each.

The hazard ratio $(95 \% \mathrm{CI})$ for disease progression in bivariate analyses was $0.79(0.62-1.01 ; \mathrm{p}=0.059)$ for angiotensin-converting enzyme inhibitor treatment, $0.91(0.76-1.08 ; \mathrm{p}=0.272)$ for statins, $1.00(0.84-1.18$; $\mathrm{p}=0.958)$ for PPI, $1.13(0.94-1.34 ; \mathrm{p}=0.192)$ for obstructive airway medications, $1.14(0.78-1.65 ; \mathrm{p}=0.505)$ for metformin, $1.07(0.87-1.31 ; \mathrm{p}=0.527)$ for diabetes medications, $1.09(0.87-1.36 ; \mathrm{p}=0.458)$ for angiotensin II receptor blockers (ARBs) and $1.14(0.76-1.72 ; \mathrm{p}=0.534)$ for anticoagulants.

Multivariable analyses explored potential interactions between pairwise combinations of concomitant medications and their association with disease progression in the overall population (figure 1). Out of 78 pairwise combinations of drugs/drug classes analysed, five suggested potentially decreased or increased risk of disease progression based on hazard ratios for the interaction terms in the models: metformin and obstructive airway medications (HR 0.30, 95\% CI 0.14-0.63), anti-inflammatory and obstructive airway medications (1.63, 1.09-2.42), ARBs and diabetes medications (1.71, 1.04-2.80), diabetes and thyroid medications (1.84, 1.01-3.35) and PPI and metformin $(2.25,1.06-4.80)$. None of the most frequently reported pairwise combinations, including statins and antithrombotics (25.3\% of patients), PPIs and antithrombotics (20.6\%), statins and PPIs (18.8\%), PPIs and obstructive airway medications (16.4\%), obstructive airway medications and antithrombotics (15.4\%) and $\beta$-blockers and antithrombotics (15.4\%), were associated with differences in IPF outcomes.

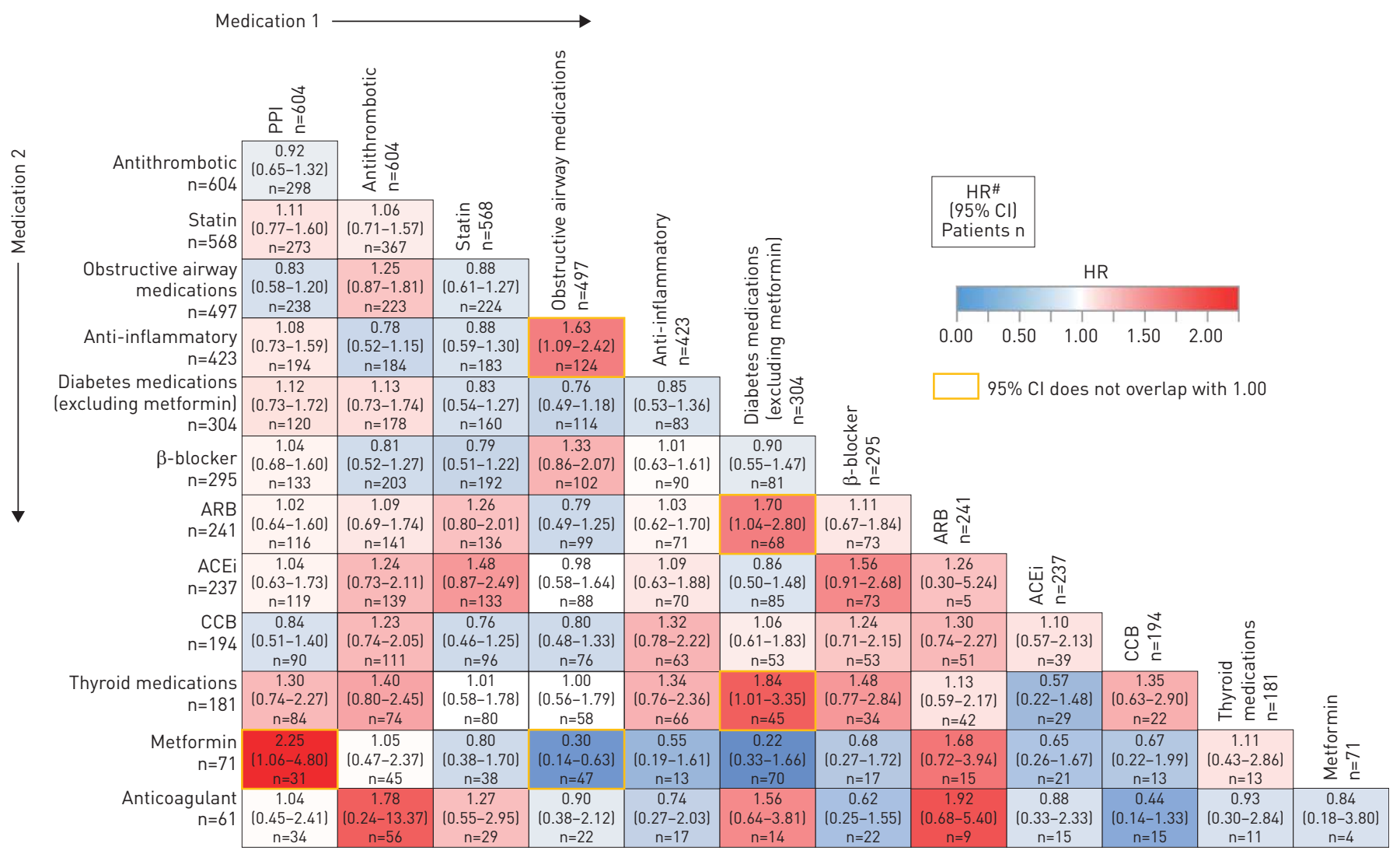

FIGURE 1 Multivariable models of concomitant medication use and disease progression results for interactions between pairwise combinations of concomitant medications. Disease progression was defined as the first occurrence of absolute decline in forced vital capacity $\geqslant 10 \%$ predicted, decline in 6-min walk distance $\geqslant 50 \mathrm{~m}$ or death from any cause over 52 weeks. ARB: angiotensin II receptor blocker; ACEi: angiotensin-converting enzyme inhibitor; CCB: calcium channel blocker; PPI: proton pump inhibitor; HR: hazard ratio. \#: adjusted for baseline demographics and clinical characteristics. HRs correspond to interaction terms (i.e. patients receiving both medication 1 and medication 2 versus patients receiving only medication 1, only medication 2 or neither). Patients missing baseline information ( $n=55)$ were excluded from multivariable analyses. 
Disease progression was observed in $62(40.5 \%)$ patients receiving no medication, 266 (35.3\%) patients receiving one to three medications and 205 (37.8\%) patients receiving four or more medications. The adjusted hazard ratio $(95 \% \mathrm{CI})$ for disease progression was $0.908(0.680-1.213$; $\mathrm{p}=0.51)$ in patients receiving one to three versus no medications and $1.063(0.779-1.450 ; \mathrm{p}=0.70)$ in patients receiving four or more versus no medications. Meaningful differences in the primary outcome were not observed when patients were analysed with more-granular categories for number of medications $(0,1-2,3-4,5-6,7-8$ and 9-10).

In the absence of data from large registries of patients with IPF, these post hoc analyses of data from phase III RCTs in patients with IPF highlight heterogeneity in concomitant medication use and comorbidities that are prevalent in the IPF population. However, these analyses found no association between the number of concomitant medications used and IPF progression. In these data, interactions between pairwise combinations of medications were associated with a broad range of HRs (HR range, 0.22-2.25), suggesting potential impacts on disease progression in patients with IPF, but the interpretation is limited.

GORD, CVD, hypertension and diabetes are frequently reported comorbidities that may impact the burden of disease in the IPF population [1, 13-15]. Use of medications that treat these comorbidities was not clearly associated with an increased risk of disease progression in this analysis, whether the medications were evaluated alone or in pairwise combinations. The exceptions to this observation were pairwise combinations of PPI and metformin, diabetes and thyroid medications, ARBs and diabetes medications and obstructive airway and anti-inflammatory medications (HR range 1.63-2.25). The findings were mostly consistent with previous analyses that found no association between treatment with antacids, statins, metformin, anticoagulants or angiotensin modulators and disease progression, although elevated mortality risk was observed in patients treated with anticoagulants or ARBs, and decreased mortality risk was observed in patients treated with statins [3-7].

Importantly, we stress that the interpretation of these findings is limited by the post hoc nature of the analyses. Moreover, medication use was evaluated only at baseline, and thus some patients may have been misclassified at the time of disease progression. Drug dose and duration of use were not evaluated. Some analyses may have been underpowered to detect differences, as many combinations of medications were reported in small numbers of patients. The number of comparisons and hypotheses tested increased the likelihood of false positives. Furthermore, these findings may not be applicable to real-world populations of patients with IPF, who often have a greater burden of comorbidities and poorer overall health.

Currently, there is a lack of evidence on the safety and impact of combinations of common medications in the IPF population. Furthermore, whether combinations of common medications affect IPF progression in patients receiving antifibrotic therapy remains an open question. These questions should continue to be examined in prospective registry studies and RCTs.

Michael Kreuter ${ }^{1}$, David J. Lederer $\odot^{2}$, Vincent Cottin $\oplus^{3}$, Nicolas Kahn ${ }^{1}$, Brett Ley ${ }^{4}$, Carlo Vancheri ${ }^{5}$, Derek Weycker $\oplus^{6}$, Mark Atwood ${ }^{6}$, Klaus-Uwe Kirchgaessler ${ }^{7}$ and Christopher J. Ryerson ${ }^{8}$

${ }^{1}$ Center for Interstitial and Rare Lung Diseases, Pneumonology, Thoraxklinik, University of Heidelberg, Member of the German Center for Lung Research, Heidelberg, Germany. ${ }^{2}$ Depts of Medicine and Epidemiology, Columbia University Irving Medical Center, New York, NY, USA. ${ }^{3}$ Dept of Respiratory Medicine, Reference Center for Rare Pulmonary Diseases, Louis Pradel Hospital, Claude Bernard University Lyon 1, UMR754, Lyon, France. ${ }^{4}$ Dept of Medicine, University of California, San Francisco, San Francisco, CA, USA. ${ }^{5}$ Dept of Clinical and Experimental Medicine, University of Catania, Catania, Italy. ${ }^{6}$ Policy Analysis Inc. (PAI), Brookline, MA, USA. ${ }^{7}$ F. Hoffmann-La Roche Ltd, Basel, Switzerland. ${ }^{8}$ Dept of Medicine, University of British Columbia, Vancouver, BC, Canada.

Correspondence: Michael Kreuter, Center for Interstitial and Rare Lung Disease, Thoraxklinik, Heidelberg University Hospital, Röntgenstraße 1, 69120 Heidelberg, Germany. E-mail: michael.kreuter@med.uni-heidelberg.de

Received: 17 June 2019 | Accepted after revision: 27 Aug 2019

This is a post hoc analysis of data from the following previous trials: NCT01366209, NCT00287716, NCT00287729 and NCT00075998. Qualified researchers may request access to individual patient-level data through the clinical study data request platform (www.clinicalstudydatarequest.com). Further details on Roche's criteria for eligible studies are available here (https://clinicalstudydatarequest.com/Study-Sponsors/Study-Sponsors-Roche.aspx). For further details on Roche's global policy on the sharing of clinical information and how to request access to related clinical study documents, see here (www.roche.com/research_and_development/who_we_are_how_we_work/clinical_trials/our_commitment_to_data_ sharing.htm).

Support statement: This manuscript was sponsored by F. Hoffmann-La Roche Ltd and Genentech, Inc. Support for third-party writing assistance, furnished by Benjamin Ricca of Health Interactions, Inc., was provided by F. Hoffmann-La Roche Ltd. Funding information for this article has been deposited with the Crossref Funder Registry.

Conflict of interest: M. Kreuter reports grants and personal fees from Boehringer Ingelheim, Galapagos and Roche, during the conduct of the study. D.J. Lederer reports personal fees from Fibrogen, Galapagos, Roche, Global Blood Therapeutics, Sanofi Genzyme and Veracyte, during the conduct of the study. V. Cottin reports grants and personal fees 
from Boehringer Ingelheim and Roche, personal fees from Actelion, Bayer, Celgene, Galapagos, Gilead, GlaxoSmithKline, Merck Sharp and Dohme, Novartis, Promedior and Sanofi, during the conduct of the study. N. Kahn reports grants and personal fees from Boehringer Ingelheim and Roche, during the conduct of the study. B. Ley reports personal fees from Roche/Genentech, during the conduct of the study. C. Vancheri reports grants and personal fees from Boehringer Ingelheim and Roche, and personal fees from Chiesi Farmaceutici, during the conduct of the study. D. Weycker is an employee of Policy Analysis Inc. (PAI). M. Atwood is an employee of Policy Analysis Inc. (PAI). K-U. Kirchgaessler is an employee of F. Hoffmann-La Roche Ltd. C.J. Ryerson reports grants and personal fees from Boehringer Ingelheim and Roche, during the conduct of the study.

\section{References}

1 Kreuter M, Ehlers-Tenenbaum S, Palmowski K, et al. Impact of comorbidities on mortality in patients with idiopathic pulmonary fibrosis. PLoS One 2016; 11: e0151425.

2 Raghu G, Rochwerg B, Zhang Y, et al. An official ATS/ERS/JRS/ALAT clinical practice guideline: treatment of idiopathic pulmonary fibrosis. An update of the 2011 clinical practice guideline. Am J Respir Crit Care Med 2015; 192: e3-e19.

3 Kreuter M, Wuyts W, Renzoni E, et al. Antacid therapy and disease outcomes in idiopathic pulmonary fibrosis: a pooled analysis. Lancet Respir Med 2016; 4: 381-389.

4 Kreuter M, Bonella F, Maher TM, et al. Effect of statins on disease-related outcomes in patients with idiopathic pulmonary fibrosis. Thorax 2017; 72: 148-153.

5 Kreuter M, Lederer DJ, Molina-Molina M, et al. Association of angiotensin modulators with the course of idiopathic pulmonary fibrosis. Chest 2019; 156: 706-714.

6 Spagnolo P, Kreuter M, Maher TM, et al. Metformin does not affect clinically relevant outcomes in patients with idiopathic pulmonary fibrosis. Respiration 2018; 96: 314-322.

7 Kreuter M, Wijsenbeek MS, Vasakova M, et al. Unfavourable effects of medically indicated oral anticoagulants on survival in idiopathic pulmonary fibrosis. Eur Respir J 2016; 47: 1776-1784.

8 Kreuter M, Costabel U, Richeldi L, et al. Statin therapy and outcomes in trials of nintedanib in idiopathic pulmonary fibrosis. Respiration 2018; 95: 317-326.

9 Costabel U, Behr J, Crestani B, et al. Anti-acid therapy in idiopathic pulmonary fibrosis: insights from the INPULSIS ${ }^{\circledR}$ trials. Respir Res 2018; 19: 167.

10 King TE J, Bradford WZ, Castro-Bernardini S, et al. A phase 3 trial of pirfenidone in patients with idiopathic pulmonary fibrosis. N Engl J Med 2014; 370: 2083-2092.

11 Noble PW, Albera C, Bradford WZ, et al. Pirfenidone in patients with idiopathic pulmonary fibrosis (CAPACITY): two randomised trials. Lancet 2011; 377: 1760-1769.

12 King TE Jr, Albera C, Bradford WZ, et al. Effect of interferon gamma-1b on survival in patients with idiopathic pulmonary fibrosis (INSPIRE): a multicentre, randomised, placebo-controlled trial. Lancet 2009; 374: 222-228.

13 Raghu G, Freudenberger TD, Yang S, et al. High prevalence of abnormal acid gastro-oesophageal reflux in idiopathic pulmonary fibrosis. Eur Respir J 2006; 27: 136-142.

14 Nathan SD, Basavaraj A, Reichner C, et al. Prevalence and impact of coronary artery disease in idiopathic pulmonary fibrosis. Respir Med 2010; 104: 1035-1041.

15 Glassberg M, Nathan SD, Lin C, et al. Cardiovascular risks, bleeding risks, and clinical events from 3 phase III trials of pirfenidone in paitents with idiopathic pulmonary fibrosis. Adv Ther 2019; 36: 2910-2926. 